\title{
Chronic Obstructive Pulmonary Disease and Obstructive Sleep Apnea. Association, consequences and treatment
}

\author{
C. Pronzato
}

\begin{abstract}
Chronic Obstructive Pulmonary Disease and Obstructive Sleep Apnea. Association, consequences and treatment. C. Pronzato.

Obstructive sleep apnea syndrome (OSAS) and chronic obstructive pulmonary disease (COPD) are two diseases that often co-exist within an individual. This co-existence, known as overlap syndrome (OS), is the result of chance rather than a pathophysiological linkage and epidemiological studies indicate a prevalence of $1 \%$ in adult males. Patients vith OS have a more important sleep-related $\mathrm{O2}$ desaturation than COPD patients with the same degree of bronchial obstruction and show an increased risk of developing hypercapnic respiratory
\end{abstract}

\begin{abstract}
failure and pulmonary hypertension when compared with patients affected by only one of he diseases. COPD and OSAS are independent risk factors for cardiovascular events and their co-existence in OS probably increases this risk. Evidence of systemic inflammation in COPD and sleep apnea and consequentely $O S$, is interesting because it may contribute to the pathogenesis of cardiovascular diseases. Treatment consists of continuous positive airway pressure (CPAP) or non-invasive positive pressure ventilation (NIPPV), with or without associated $\mathrm{O}_{2}$, for correction of the upper airway obstructive episodes and hypoxemia during sleep.

Monaldi Arch Chest Dis 2010; 73: 4, 155-161.
\end{abstract}

Keywords: Chronic obstructive pulmonary disease, Sleep apnea-hypopnea syndrome, Inflammation, Cardiovascular disease, Noninvasive ventilation.

U.O. di Pneumologia Riabilitativa, Istituto Scientifico di Montescano IRCCS, Fondazione Salvatore Maugeri - Montescano (Pavia), Italy.

\section{Introduction}

Chronic obstructive pulmonary disease (COPD) and obstructive sleep apnea syndrome (OSAS) represent two of the most prevalent chronic respiratory disorders in clinical practice. A possible association between these diseases was described in the mid1980s by Flenley [1], who named this association "overlap syndrome". COPD is presently defined by the presence of an obstructive ventilatory defect characterised by a $\mathrm{FEV}_{1} / \mathrm{FVC}$ ratio less than $70 \%$ in patients with no other chronic respiratory disease [2]. As regards OSAS, it is characterised by repetitive episodes of complete or partial upper airway obstruction that occur during sleep and daytime consequences which include subjective daytime sleepiness and impaired cognitive function, including impaired memory. The diagnostic criteria of OSAS must fulfill both subjective symptoms and five or more obstructive breathing events documented by overnight monitoring [3].

\section{Epidemiological considerations}

\section{Epidemiology}

COPD is the most common chronic lung disease, since several epidemiological studies have shown that about $8 \%$ to $10 \%$ of the population above 30 years of age in developed countries is affected by this disorder [2]. The prevalence of OSAS is also very high and a comprehensive review and analysis of available data by Young and coll. places the prevalence at $5 \%$ of adults in developed countries [4]. Considering the high prevalence of these two diseases, the presence of both of these conditions in the same subjects is not a rare occurance. Early reports found a high prevalence of OSAS in patients with COPD or conversely a high prevalence of chronic airways obstruction in OSA patients [5-6]. However, a study conducted on 1,132 participants using data of the Sleep Heart Health Study, found a similar prevalence of OSAS in patients with COPD as in an equivalent general population [7]. Results indicate that the respiratory disturbante index (RDI) was less in subjects with lower airway obstruction, largely as a consequence of lower body mass index (BMI), because RDI values were similar when stratified for BMI. More recently, data from an epidemiological study performed in Poland on middle-aged and elderly subjects [8], show that OSAS prevalence was relatively high (11.3\%) and overlap syndrome occurred in $9.2 \%$ of OSAS population and $1 \%$ of total population. Both trials confirmed the hypothesis that the coexistence of COPD and OSAS is due to chance rather through a pathophysiologic linkage between the two conditions.

\section{Pathophysiological interactions of COPD and OSAS}

The relationship between COPD and OSAS may be influenced by several factors. Body mass 
and smoking may affect pathophysiological relationships. Neck obesity contributes to upper airways narrowing [9], truncal obesity promotes ventilatory disturbance by reduced chest wall compliance and respiratory muscle strenght [10] and is also associated with reduced FRC, which contributes to ventilation-perfusion mismatching. However low BMI is common in COPD, especially in patients suffering in the advanced stages of the disease, and may protects against OSAS: this hypothesis is supported by the finding of a lower RDI in subjects with airways obstruction, relating to a lower BMI [7]. Smoking is a well known risk factor for COPD and predisposes to sleep apnea by increasing upper airway resistence due to local inflammation and edema [9]. Redolfi and coll. in a study on healthy subjects, demonstrated an overnight rostral fluid displacement from the legs, causing a growing in the circumference of the neck [11]. In COPD patients, often affected by cor pulmonale, rostral shift of peripheral edema when supine might result in fluid accumulation in the neck, contributing to pharyngeal narrowing and favouring the onset of obstructive apneas and hypopneas.

\section{Quality of sleep}

Many patients with severe COPD and marked daytime hypoxemia complain of poor-quality sleep and the most frequent polysomnographic findings in these subjects are a decreased total sleep time, delayed sleep onset, reduction in REM sleep and more changes in sleep stage [12]. This sleep disorganisation may be related to nocturnal cough and dyspnea, alterations in gas exchange, medications or general debility associated with COPD [13-14]. On the other hand, the study of Sanders and coll. [7] where 1132 participants with mild COPD were investigated, has shown that in the absence of sleep apnea, sleep was minimally perturbed: there were statistically significant but small differences between patients who did or did not have COPD with regard to total sleep time; no differences were observed with regard to Epworth Sleepiness Score (ESS), sleep latency, sleep efficency, arousal index or percent of total sleep time (TST) spent in individual sleep stages. Subjects with overlap, compared with the others only affected by obstructive airways disease, had higher ESS, higher arousal index, lower total sleep time and lower sleep efficency. Only small differences were found between patients with OSAS alone and those with both disorders, therefore sleep quality in patients with overlap is mostly influenced by the presence of OSAS.

\section{Blood gases and pulmonary function features}

In a recent metaanalysis [15] daytime hypercapnia in obese patients with OSAS was associated with severity of OSA, higher BMI levels and degree of restrictive chest wall mechanics. Hypoxemia is also described in OSA and hypoxemic events in OSAS patients are closely associated with apneas and hypopneas and result from alveolar hypoventilation. Worsening hypoxemia, seen at night-time in COPD patients, is attributable to a combination of ventilation-perfusion mismatching and alveolar hypoventilation, the second representing the predominant mechanism, especially during REM sleep [16] in which hypoventilation is quite common also in normal controls. As many such patients have awake hypoxemia, they are especially prone to nocturnal oxygen desaturation by being on the steep portion of the oxyhemoglobin dissociation curve. In addition, they have the disadvantage of increased tidal volume due to flattened diaphragms and diminished respiratory drive, demostrating a more pronounced hypercapnic response during sleep [17].

The overlap syndrome is said to predispose to daytime hypercapnia and hypoxemia independently of lung function [18]. OSA seems to be an important cause of hypoxemia and hypercapnia in some groups of patients in whom these findings appear to be disproportionate to the level of lung function impairment [6-19]. Chan et al. demonstrated that hypercapnic COPD patients had many more sleep-disordered breathing events, higher BMI and smaller upper airways cross-sectional areas when compared with eucapnic controls matched for lung function [20].

Furthermore it is known that overlap patients present more nocturnal desaturation than patients with either OSAS or COPD alone [6-7]. Sanders and coll. [7] examined the degree to which COPD and OSAS independently and jointly contribute to desaturation during sleep. After adjusting for confounding factors, the odd ratio (OD) for oxyhemoglobin saturation below 90 and $85 \%$ for more than $5 \%$ of TST was 20-times greater in individuals with OSAS alone compared with those who did not suffer with either disorder and 30-times greater in participants with both disorders (overlap patients). Bednarek and coll. [8] demonstrated that overlap individuals had night-time lower mean arterial blood saturation and spent more time in desaturation than the OSAS group.

Very recently Chien and coll. showed that repetitive inspiratory effort against an obstructed airway and intermittent hypoxia may be deleterious to the inspiratory muscles in patients with severe OSA. A significantly lower functional performance was shown for both inspiratory muscles and peripheral ones in a group of OSAS patients compared with healthy controls. However, higher fatigability was only seen in the inspiratory muscles of patients with severe OSA [21].

\section{Inflammation}

Low-intensity chronic systemic inflammation has been found in patients with COPD as well as in those with OSA. However, no studies have investigated the interaction of both these diseases in the development of systemic inflammation. It is possible that the concomitance of COPD and OSA may worsen these alterations, inducing a more rapid or severe development of cardio-vascular 
impairment. Up to now only indirect data supports this hypothesis. Marin and coll. found that patients with concomitant COPD and untreated OSA died more frequently for cardiovascular events than patients with concomitant COPD and treated OSA or patients with COPD alone [22].

Some molecular pathways of systemic inflammation in COPD and OSA seem to be similar, suggesting a multiplicative effect in overlap syndrome. In particular, some inflammatory mediators are elevated in both OSAS and COPD, but it is difficult to discriminate possible different inflammatory profile among the different phenotypes characterising COPD.

C-reactive protein (CRP), an acute-phase inflammatory protein, contributes to atherosclerosis by promoting adhesion molecule expression. CRP levels, which correlate with future cardiovascular events [23], are elevated in OSAS [24], but obesity is a major confounding variable and the evidence of an independent relationship between OSAS and CRP levels is not clear. Some studies [25] have found no association between circulating IL-6 levels and OSAS, particularly after adjustment for BMI. On the other hand it has been observed that CRP [26] and IL-6 levels are elevated in COPD patients with stable disease and increase during exacerbations [27].

The transcription factor (nuclear factor) NF-KB is a master regulator of inflammatory gene expression and regulates cytokines such as tumour necrosis factor (TNF)- $\alpha$ and IL- 8 that contribute to atherosclerosis by inducing adhesion molecules expression [28]. NF-KB activation is induced by hypoxia, which also causes activation of the adaptive transcription factor, hypoxia-inducible factor (HIF)-1, a molecule regulating many genes which promote tissue perfusion and oxygenation. In a recent 'in vitro' study Ryan and coll. [29] demonstrated a preferential activation of NF-KB during intermittent hypoxia whereas sustained hypoxia resulted in preferential activation of HIF-1. Given that intermittent hypoxia is the prevalent hypoxic pattern in OSAS, these observations allow some fascinating hypotheses, that of course require 'in vivo' investigations.

Circulating TNF- $\alpha$ and IL-8 levels correlate with early atherosclerosis and are predictive of coronary heart disease and congestive heart failure [30]. In both COPD and OSAS TNF- $\alpha$ and IL-8 levels are elevated if compared with control subjects, and this appears to be independent of obesity [31]. In COPD patients with low body weight, TNF$\alpha$ elevation is associated with muscle wasting, probably due to its accumulation in muscle tissue [32].

Furthermore oxidative stress occurs in COPD [33] and OSAS [34] and is associated with an increase in reactive oxygen species (ROS) production, principally from intrapulmonary leukocytes in COPD [33] and circulating leukocytes in OSAS [35]. Although ROS serve important physiological roles in signal transduction, excessive production may damage cellular components and lead to oxidation of macromolecules, including lipids, proteins and DNA [36], contributing to vascular endothelial dysfunction.
Finally the presence of activation and/or dysfunction of circulating leukocytes in COPD and OSAS has particular relevance because leukocytes accumulation. In addition their adhesion to the endothelium is of key importance in atherosclerotic plaque formation [28]. A systematic meta-analysis has shown that circulating neutrophil numbers are elevated in COPD [26] and an 'in vivo' study reports abnormalities of these cells, such as impaired neutrophil apoptosis and increased expression of surface adhesion molecules in OSAS patients [37].

\section{Cardiovascular disease}

The mechanisms leading to the onset of cardiovascular disease in COPD and OSAS are multifactorial, principally involving systemic inflammation and arterial blood gases. Inflammation plays a key role in atherosclerotic plaque formation from developing of endothelial dysfunction in response to oxidised lipids, inflammatory cytokines such as TNF- $\alpha$, IL- 6 and other factors including ROS, to involvement of intercellular adhesion molecules, promoting rolling and adherence of leukocytes to endothelium, until the accumulation of lipids and ultimately to plaque rupture [28]. Both COPD and OSAS are associated with increased activation of many inflammatory cell and molecular mechanisms involved in atherosclerosis $[38,39]$. It provides basic mechanisms to support the clinical and epidemiological data demonstrating COPD and OSAS as independent risk factors for cardiovascular disease $[40,41]$.

Alveolar hypoxia is the most important mechanism leading to pulmonary arterial vasoconstriction and pulmonary ipertension $(\mathrm{PH})$ [42], and COPD is frequently complicated by the development of pulmonary ipertension [43]. OSAS patients may also present susteined $\mathrm{PH}$ and the risk increases considerably if it is associated with COPD, obesity or both [44]. Chaouat and coll. [6] have observed that the prevalence of $\mathrm{PH}$ was of $42 \%$ in patients with overlap, much higher than in OSAS alone (13\%). Patients with overlap can develop $\mathrm{PH}$ even if they do not exhibit a marked degree of bronchial obstruction. In COPD, PH is generally observed in severe bronchial obstruction $\left(\mathrm{FEV}_{1}<50 \%\right.$ of the predicted value and $\leq 1000 \mathrm{ml}$ ), leading to significant hypoxiemia. In overlap patients investigated by Fletcher and coll. $\mathrm{FEV}_{1} / \mathrm{FVC}$ was close to $60 \%$, contrasting with marked hypoxiemia and $\mathrm{PH}$ [45]. This can be explained by the synergistic effects of the diseases on pulmonary hemodynamics and also on gas exchange. In fact in COPD patients, $\mathrm{PH}$ is frequently observed when daytime $\mathrm{PaO}_{2}$ is less than 55 to $60 \mathrm{mmHg}$ [46]. The average daytime $\mathrm{PaO}_{2}$ of the overlap patients in the study by Chaouat previosly quoted was higher $(66 \pm 10 \mathrm{mmHg})$ but the mean $\mathrm{PaO}_{2}$ during sleep is certainly lower because of the ripetition of apneas and hypopneas. Thus, nocturnal oxygen desaturation is greater in overlap patients than in COPD or OSAS alone [7], apnea-associated desaturation is more pronounced and daytime hypercapnia also more common. The combination of 
more pronounced hypoxiemia and hypercapnia might result in greater cardiovascular morbidity.

About cardiac rhythm disturbances associated with OSAS and COPD, premature ventricular contractions have been observed commonly during sleep in COPD patients with nocturnal $\mathrm{SaO}_{2}$ less than $80 \%$ [47]. In patients with OSAS the entire spectrum of cardiac arrhythmias has been observed [48]; the most common abnormality, present in the majority of patients with severe OSAS, is marked sinus arrhytmia, characterised by reduction of heart rate during apnea, followed by increase of heart rate on resumption of respiration. Finally, Olmetti and coll. [49] found that tachyarrhythmia is more common in patients with OSAS and concomitant COPD, in particular those taking long-acting $\beta_{2}$-stimulants, than in patients with OSA alone.

Lavie and coll. showed that OSA patients who smoke currently have higher levels of biochemical cardiovascular risk markers than non-smokers [50]. We can suppose that smoke, OSA and COPD may represent a cluster that identifies high risk subjects for premature death, particularly for cardiovascular diseases. Cross-sectional studies may have failed to find statistically significant association among these factors only for a sort of "mortality effect". On the other hand this hypothesis seems to be confirmed by data collated by Marin and coll. [22] that found an increasing risk of cardiovascular deaths in COPD patients with concomitant OSA.

\section{Evaluation}

Testing for sleep apnea is not necessary in patients who have COPD, but overnight oximetry should be considered in most COPD patients to identify significant overnight desaturation, and clinical assessment should include question about sleep quality and possible co-existing OSAS. COPD individuals who possess typical risk factors for OSA, such as obesity, chronic snoring, enlarged neck, daytime sleepiness and hypertension, but also presenting nocturnal hypoxiemia complications unexplained by waking arterial oxigen levels and pulmonary hypertension that is out of proportion to the severity of pulmonary function derangement [51] or neuropsychologic impairments, should be evaluated according to standard screening practices. The most appropriate method for diagnosis of the overlap syndrome continues to be routine polysomnigraphy because nocturnal oximetry is not able to detect those patients with subtle sleep-disordered breathing, showing frequent apneas and hypopneas without desaturation, but significant sleep disruption.

\section{Treatment}

Treatment of the overlap syndrome is based on the patient and on the severity of the disease and considerations on co-existing illnesses, including obesity, heart failure or secondary pulmonary hypertension, should also be made. Furthermore, pa- tients should be advised of the importance of avoiding factors that increase the severity of upper-airway obstruction such as sleep deprivation, alcohol assumption, hypnotic drugs, or increased weight. Treatment options may include oxygen, oral appliance, continuous positive airway pressure (CPAP) and non-invasive positive pressure ventilation (NIPPV). Auto-titrating CPAP is not currently recommended for individuals affected by COPD [52].

Patients with COPD and mild sleep-disordered breathing but significant nocturnal hypoxiemia may poorly tolerate CPAP and be better treated with oxygen and optimising medical management of airway obstruction. However, considering that most oxygen studies $[18,53]$ were performed before the widespread use of polysomnigraphy, further evaluation of the benefits of oxygen therapy in patients with COPD and minimal sleep-disordered breathing or non tolerance of CPAP would be necessary.

In individuals with more severe forms of sleep-disordered breathing, treatment with CPAP is effective in improving RDI, nocturnal hypoxemia and hypercapnia and daytime sleepiness. In overlap patients the benefit of CPAP may arise from improvement in respiratory mechanics, such as reducing the work of breathing by minimiaing hyperinflation. Two previous studies have shown that the use of CPAP improves lung function $\left(\mathrm{FEV}_{1}, \mathrm{FVC}\right)$, gas exchange and respiratory muscle function in patients with coexisting COPD and OSAS $[54,55]$.

Until now, benefits of long-term treatment with CPAP and in particular its impact on survival in the overlap syndrome were unknown. Two studies, recently concluded, assessed the effects of CPAP on survival and hospitalisation of these patients. Machado and coll., in an observational study, evaluated 95 patients with moderate-to-severe OSAS associated with hypoxaemic COPD; the study cohort was hypoxaemic and hypercapnic at rest, received long-term oxygen therapy (LTOT) and showed severe airflow obstruction. CPAP treatment was performed in 61 individuals, the remainders 34 being not adherent to ventilatory treatment. The 5-yr survival estimate was $71 \%$ and $26 \%$ in the CPAP-treated and non-treated groups, respectively. After adjusting for confounding factors, patients treated with CPAP showed a significantly lower risk of death [56]. Furthermore, Marin and coll. studied 441 patients with overlap syndrome, 228 treated with CPAP and 213 not treated, and 210 patients with COPD without OSA, for about 10 years. Results show principally that the co-existence of COPD and OSAS is associated with increased mortality compared with COPD alone, in particular a significant higher number of cardiovascular deaths was observed. Secondly, an effective CPAP treatment of OSA reduces mortality in overlap patients. Finally, patients with overlap syndrome not-treated with CPAP were more likely to suffer a severe COPD exacerbation leading to hospitalization versus the COPD-only group, but the risk is decreased by CPAP treatment [22]. 
In patients that showed clear signs and symptoms of nocturnal hypoxia or hypoventilation, CPAP may be inefficient, particularly during REM sleep. Consequently it may be necessary to add supplementary $\mathrm{O}_{2}$ when the mean nocturnal $\mathrm{SaO}_{2}$ under CPAP alone is less than $90 \%$ or to shift to NIPPV that is finalised to correct hypoventilation. In patients with severe hypercapnic COPD without OSA, the long-term effects of NIPPV are controversial, whereas results are excellent in the obesity-hypoventilation syndrome. At present there have been no perspective studies that have compared CPAP with non-invasive mechanical ventilation in patients with overlap syndrome.

\section{Open points}

The new definition of OSA proposed by AASM in 2007 [57] based on epidemiological data which appeared in SHHS determines a significant change of perspective. Hence the data of OSA prevalence is completely different from the past, therefore data relevant to the relationship between OSA and other chronic diseases should be re-evaluated. In particular, it is necessary to redefine the prevalence of overlap syndrome in general population and COPD population. This data is especially important to define new patients phenotype as well as interactions among several levels of severity of these two pathologies in the clinical evolution of the overlap syndrome and interactions of therapeutical treatments on patient prognosis. As already suggested new "physiological" studies are needed to understand cellular and humoral mechanisms through which overlap syndrome determines its effect. It seems in fact that from the clinical and epidemiological point of view these effects are greater than the sum of the two pathologies.

Sleep apnea itself has been found to determine daytime hypoxemia [58]. Several papers published in the past $[6,18]$ have shown that patients with overlap syndrome have an amount of daytime hypoxemia higher than what could have been expected on the basis of lung mechanics derangement. The effect of CPAP therapy on daytime $\mathrm{PaO}_{2}$ in patients with overlap syndrome has not been investigated. In the paper of Machado [56], above mentioned, seems that some indications of LTOT should be reversed in a group of patients. This data should be confirmed in a perspective study.

Finally the introduction of a more standardised therapeutical approach is now mandatory. CPAP therapy is the first choice therapy for patients with a phenotype "OSA prevalent". However, we are confident that CPAP therapy is not as safe in patients with COPD as is generally supposed. Indeed, recently Holanda and coll. found that CPAP in COPD patients increased lung volume worsening the baseline level of alveolar hyperinflation [59]. Non-invasive mechanical ventilation has been proposed by AASM guidelines for treatment of patients with overlap syndrome, especially for those CPAP intolerant or with severe nocturnal hypoxia and/or hypoventilation. Pressure support ventilation has been proposed by AASM to sta- bilise upper airways in patients with OSA or overlap syndrome for the possibility to change the level of effective pressure between inspiration and expiration. Pressure support ventilation is now the most common mode of ventilation proposed for treatment of nocturnal hypoventilation in COPD patients.

\section{References}

1. Flenley DC. Sleep in chronic obstructive pulmonary disease. Clin Chest Med 1985; 6: 651-661.

2. Pauwels RA, Buist AS, Calverly PM, et al. Global strategy for the diagnosis, management, and prevention of chronic obstructive pulmonary disease: NHLBI/WHO Global Initiative for Chronic Obstructive Lung Disease (GOLD) Workshop summary. Am J Respir Crit Care Med 2001; 163: 1256-1276.

3. Banno K, Kryger MH. Sleep apnea: Clinical investigations in humans. Sleep Med 2007; 8: 400-426.

4. Young T, Peppard PE, Gottlieb DJ. Epidemiology of obstructive sleep apnea. Am J Respir Crit Care Med 2002; 165: 1217-1239.

5. Guilleminault C, Cummiskey J, Motta J. Chronic obstructive airflow disease and sleep studies. Am Rev Respir Dis 1980; 122: 397-406.

6. Chaouat A, Weitzemblum E, Krieger J, Ifoundza T, Oswald M, Kessler R. Association of chronic obstructive pulmonary disease and sleep apnea syndrome. Am Rev Respir Dis 1995; 151: 82-86.

7. Sanders MH, Newman AB, Haggerty CL, et al. Sleep and sleep-disordered breathing in adult with predominantly mild ostructive airway disease. Am J Respir Crit Care Med 2003; 167: 7-14.

8. Bednarek M, Plywaczewski R, Jonczak L, Zielinski J. There is no relationship between chronic obstructive pulmonary disease and obstructive sleep apnea syndrome: a population study. Respiration 2005; 72: 142-149.

9. Deegan PC, McNicholas WT. Pathophysiology of obstructive sleep apnoea. Eur Respir J 1995; 8: 11611178.

10. Poulain M, Doucet M, Major GC, et al. The effect of obesity on chronic respiratory diseases: pathophysiology and therapeutic strategies. Can Med Assoc J 2006; 174: 1293-1299.

11. Redolfi S, Yumino D, Ruttanaumpawan P, et al. Relationship between overnight rostral fluid shift and obstructive sleep apnea in nonobese men. Am J Respir Crit Care Med 2009; 179: 241-246.

12. Breslin E, Van der Schans C, Breubink S, et al. Perception of fatigue and quality of life in patients with COPD. Chest 1998; 114: 958-964.

13. Cormick W, Olson LG, Hensley MJ, Saunders NA. Nocturnal hypoxaemia and quality of sleep in patients with chronic obstructive lung disease. Thorax 1986; 41: 846-854.

14. Mulloy E, McNicholas WT. Theophylline improves gas exchange during rest, exercise and sleep in severe chronic obstructive pulmonary disease. Am Rev Respir Dis 1993; 148: 1030-1036.

15. Kaw R, Hernandez AV, Walker E, Aboussouan L, Mokhlesi B. Determinants of hypercapnia in obese patients with obstructive sleep apnea: a systematic review and metaanalysis of cohort studies. Chest 2009; 136: 787-796.

16. Becker HF, Piper AJ, Flynn WE, et al. Breathing during sleep in patients with nocturnal desaturation. Am J Respir Crit Care Med 1999; 159: 112-118.

17. Hiestand D, Phillips B. The overlap syndrome: chronic obstructive pulmonary disease and obstructive sleep apnea. Crit Care Clin 2008; 24: 551-563. 
18. Chaouat A, Weitzemblum E, Kessler R, et al. Sleep-related $\mathrm{O} 2$ desaturation and daytime pulmonary haemodynamics in COPD patients with mild hypoxaemia. Eur Respir J 1997; 10: 1730-1735.

19. Berthon-Jones M, Sullivan CE. Time course of change in ventilatory response to $\mathrm{CO} 2$ with long-term CPAP therapy for obstructive sleep apnea. Am Rev Respir Dis 1987; 135: 144-147.

20. Chan CS, Bye PTP, Woolcock AJ, Sullivan CE. Eucapnia and hypercapnia in patients with chronic airflow limitation. The role of the upper airways. Am Rev Respir Dis 1990; 141: 861-865.

21. Chien MY, Wu YT, Lee PL, Chang YJ, Yang PC. Inspiratory muscle dysfunction in patients with severe obstructive sleep apnoea. Eur Respir J 2010; 35: 373-380.

22. Marin JM, Soriano JB, Carrizo SJ, Boldova A, Celli BR. Outcomes in patients with chronic obstructive pulmonary disease and obstructive sleep apnea. The overlap syndrome. Am J Respir Crit Care Med 2010; 172: 189-194.

23. Ridker PM, Cushman M, Stampfer MJ, Tracy RP, Hennekens $\mathrm{CH}$. Inflammation, aspirin, and the risk of cardiovascular disease in apparently healthy men. $N$ Engl J Med 1997; 336: 973-979.

24. Yokoe T, Minoguchi K, Matsuo H, et al. Elevated levels of C-reactive protein and interleukin-6 in patients with obstructive sleep apnea syndrome are decreased by nasal continuous positive airway pressure. Circulation 2003; 107: 1129-1134.

25. Mehra R, Storfer-Isser A, Kirchner HL, et al. Soluble interleukin 6 receptor: a novel marker of moderate to severe sleep-related breathing disorders. Arch Intern Med 2006; 166: 1725-1731.

26. Gan WQ, Man SFP, Senthilselvan A, Sin DD. Association between chronic obstructive pulmonary disease and systemic inflammation: a systematic review and a meta-analysis. Thorax 2004; 59: 574-580.

27. Hurst JR, Perera WR, Wilkinson TMA, Donaldson GC, Wedzicha JA. Systemic and upper and lower airway inflammation at exacerbation of chronic obstructive pulmonary disease. Am J Respir Crit Care Med 2006; 173: 71-78.

28. Hansson GK. Inflammation, atherosclerosis, and coronary artery disease. N Engl J Med 2005; 352: 16851695.

29. Ryan S, Taylor CT, McNicholas WT, et al. Selective activation of inflammatory pathways by intermittent hypoxia in obstructive sleep apnea syndrome. Circulation 2005; 112: 2660-2667.

30. Cesari M, Penninx BWJH, Newman AB, et al. Inflammatory markers and onset of cardiovascular events: results from the Health ABC Study. Circulation 2003; 108: 2317-2322.

31. Ryan S, Taylor CT, McNicholas WT. Predictors of elevated nuclear factor $\mathrm{KB}$-dependent genes in obstructive sleep apnea syndrome. Am J Respir Crit Care Med 2006; 174: 824-830.

32. Eid AA, Ionescu AA, Nixon LS, et al. Inflammatory response and body composition in chronic obstructive pulmonary disease. Am J Respir Crit Care Med 2001; 164: 1414-1418.

33. MacNee W. Oxidants/antioxidants and COPD. Chest 2000; 117: 303S-317S.

34. Lavie L. Obstructive sleep apnoea syndrome: an oxidative stress disorder. Sleep Med Rev 2003; 7: 35-51.

35. Schulz R, Mahmoudi S, Hattar K, et al. Enhanced release of superoxide from polymorphonuclear neutrophils in obstructive sleep apnea: impact of continuous positive airway pressure therapy. Am J Respir Crit Care Med 2000; 162: 566-570.

36. Droge W. Free radicals in the physiological control of cell function. Physiol Rev 2002; 82: 47-95.
37. Dyugovskaya L, Polyakov A, Lavie P, Lavie L. Delayed neutrophil apoptosis in patients with sleep apnea. Am J Respir Crit Care Med 2008; 177: 544-554.

38. McNicholas WT, Bonsignore MR. Sleep apnoea as an independent risk factor for cardiovascular disease: current evidence, basic mechanisms and research priorities. Eur Respir J 2007; 29: 156-178.

39. MacNee W, Maclay J, McAllister D. Cardiovascular injury and repair in chronic obstructive pulmonary disease. Proc Am Thorac Soc 2008; 5: 824-833.

40. Sin DD, Man SFP. Chronic obstructive pulmonary disease as a risk factor for cardiovascular morbidity and mortality. Proc Am Thorac Soc 2005; 2: 8-11.

41. Somers VK, White DP, Amin R, et al. Sleep apnea and cardiovascular disease: an American Heart Association/American College of Cardiology Foundation scientific statement from the American Heart Association Council for High Blood Pressure Research Professional Education Committee, Council on Clinical Cardiology, Stroke Council, and Council on Cardiovascular Nursing. J Am Coll Cardiol 2008; 52: 686-717.

42. Bonsignore MR, Marrone $\mathrm{O}$, Insalaco G, et al. The cardiovascular effects of obstructive sleep apnoeas: analysis of pathogenic mechanisms. Eur Respir J 1994; 7: 786-805.

43. Chaouat A, Bugnet AS, Kadaoui N, et al. Severe pulmonary hypertension and chronic obstructive pulmonary disease. Am J Respir Crit Care Med 2005; 172: 189-194.

44. Chaouat A, Weitzenblum E, Krieger J, et al. Pulmonary hemodynamics in the obstructive sleep apnea syndrome. Chest 1996; 109: 380-386.

45. Fletcher EC, Schaal JM, Miller J, Fletcher JG. Longterm cardiopulmonary sequelae in patients with sleep apnea and chronic lung disease. Am Rev Respir Dis 1987; 135: 525-533.

46. Ashutosh K, Mead G, Dunsky M. Early effects of oxygen administration and prognosis in chronic obstructive pulmonary disease and cor pulmonale. Am Rev Respir Dis 1983; 127: 399-404.

47. Shepard JW, Garrison MW, Grither DA, et al. Relationship of ventricular ectopy to nocturnal oxygen desaturation in patients with chronic obstructive pulmonary disease. Am J Med 1985; 78: 28-34.

48. Arias MA, Sanchez AM. Obstructive sleep apnea and its relationship to cardiac arrhytmias. J Cardiovasc Electrophysiol 2007; 18: 1006-1014.

49. Olmetti F, La Rovere MT, Robbi E, Taurino AE, Fanfulla F. Nocturnal cardiac arrhythmia in patients with obstructive sleep apnea. Sleep Medicine 2008; 9: 475-480.

50. Lavie L, Lavie P. Smoking interacts with sleep apnea to increase cardiovascular risk. Sleep Medicine 2008; 9: 247-253.

51. Douglas NJ, Flenley DC. Breathing during sleep in patients with obstructive lung disease. Am Rev Respir Dis 1990; 141: 1055-1070.

52. Littner M, Hishkowitz M, Davila D, et al. Practice parameters for the use of auto-titrating continuous positive pressure devices for titrating pressure and treating adult patients with obstructive sleep apnea syndrome. An American Academy of Sleep Medicine report. Sleep 2002; 25: 143-147.

53. Chaouat A, Weitzemblum E, Kessler R, et al. A randomized trial of nocturnal oxygen therapy in chronic obstructive pulmonary disease patients. Eur Respir $J$ 1999; 14: 1002-1008

54. De Miguel J, Cabello J, Sanchez-Alarcos JM, et al. Long-term effects of treatment with nasal continuous positive airway pressure on lung function in patients with overlap syndrome. Sleep Breath 2002; 6: 3-10.

55. Mansfield D, Naughton MT. Effects of continuous positive airway pressure on lung function in patients with 
chronic obstructive pulmonary disease and sleep disordered breathing. Respirology 1999; 4: 365-370.

56. Machado MCL, Vollmer WM, Togeiro SM, et al. CPAP and survival in moderate-to-severe obstructive sleep apnoea syndrome and hypoxaemic COPD. Eur Respir J 2010; 35: 132-137.

57. Iber C, Ancoli-Israel S, Chesson AL, Quan SF eds. For the American Academy of Sleep Medicine. The AASM manual for the scoring of sleep and associated events. Rules, terminology, and technical specifications.
Westchester III, Illinois: American Academy of Sleep Medicine, 2007.

58. Fanfulla F, Grassi M, Taurino AE, D'Artavilla Lupo N, Trentin R. The relationship of daytime hypoxemia and nocturnal hypoxia in Ostructive Sleep Apnea Syndrome. Sleep 2008; 31: 249-255.

59. Holanda MA, Fortaleza SCB, Alves-de-Almeida M, et $a l$. Continuous positive ayrway pressure effects on regional lung aeration in patients with COPD: a high-resolution CT scan study. Chest 2010; 138: 305-314.

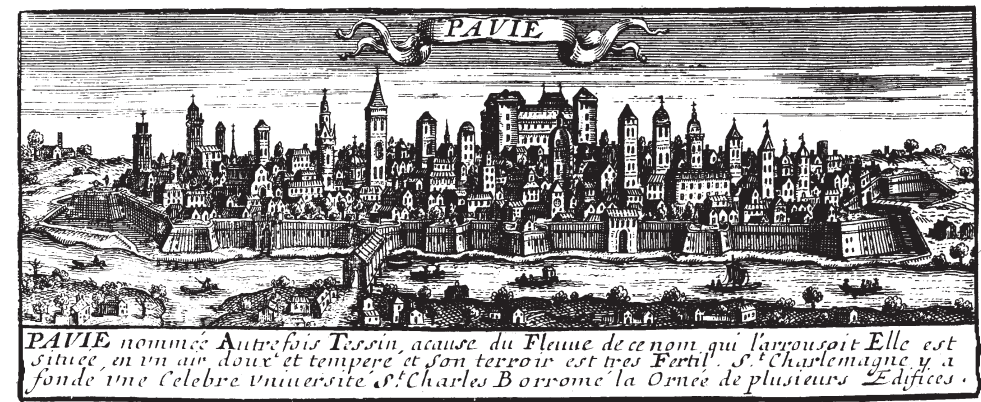

